\title{
Improved LEACH Protocol for Increasing Network Lifetime Based on Circular Patches Clustering
}

\author{
Awj R. Tarawneh, Banan Malahmeh, Abdullah Al-Odienat ${ }^{*}$ \\ Department of Electrical Engineering, Faculty of Engineering, Mutah University, Jordan \\ Received February 28, 2020; Revised April 12, 2020; Accepted April 27, 2020
}

Copyright $\odot 2020$ by authors, all rights reserved. Authors agree that this article remains permanently open access under the terms of the Creative Commons Attribution License 4.0 International License

\begin{abstract}
Wireless sensor network (WSN) plays a major role in the modern world. It is a useful technology for sending and receiving data from several parts of the system using mini sensor nodes that are distributed over a wide area. These nodes are capable of performing several data operations like sensing the environment, gathering, processing the data and other operations. They receive the essential energy for performing these processes from the batteries embedded in these nodes. In many applications, the sensor nodes are small equipped with a small size battery of little energy. It is very important to reduce energy consumption and to make the lifetime of the network as long as possible. For this purpose, the Low Energy Adaptive Clustering Hierarchy (LEACH) routing protocol has been developed. In this paper, a modified model of LEACH protocol is developed to further reduce energy consumption and consequently, increasing the lifetime of the network.
\end{abstract}

Keywords Wireless Sensor Network (WSN), Sensor Node, LEACH Protocol, Energy Consumption, Sensor Lifetime

\section{Introduction}

Wireless Sensor Networks (WSN) are decentralized networks that got significant attention for their wide scope of applications such as health, smart buildings, security, and agriculture monitoring [1]. WSN constitutes of a large number of tiny, cheap and limited battery-powered nodes that work together to perform several tasks [2]. The sensor nodes communicate with each other in the network for gathering and sending a variety of messages about the monitored object to the sink (base station), which in turn, processes the information and reports it to the end-user [3].

To accomplish these tasks, a large number of protocols are proposed in the literature. However, the main problem faces the WSN protocols, is that every node in the network has limited battery energy. Increasing network lifetime is one of the serious challenges that should be studied during the designing stage of the network. As sensor nodes drain their energy in activities such as sensing, processing, transmitting data, and communication which is the biggest energy consumer, there should be a management plan to improve the network lifetime [2].

The lifetime of the network is the time to the first sensor node consumes all its power and becomes dead [3]. Nowadays there are many kinds of low energy consuming protocols that increase the networks' lifetime, they are divided into two groups, flat and hierarchical [4]. In this paper, the hierarchical clustering type will be addressed. The clustering-based hierarchy extends the lifetime by increasing the hierarchy level based on the purpose of the application of WSN [5]. The clustering-based routing protocols can be divided into two major groups; distributed and centralized. The responsible element in the distributed clustering is the nodes while the base station is the responsible element in the case of centralized clustering to perform the data transmitting process [1].

In earlier works, many types of LEACH were studied, in [6] the Classic Leach was studied and was compared to the non-clustering algorithms where the time of the first dead node is eight times larger than the non-clustering. After that, many modifications were done to LEACH to increase the network lifetime or decrease the energy consumption, for example in [7] a centralized LEACH was developed by distributing the Clustering Hierarchy $(\mathrm{CH})$ nodes all over the network. However, its problem was the need for Global Positioning System (GPS) to communicate with the base station and that would increase energy consumption.

Another modification was presented by M. Tong in [8] called LEACH-B which refers to balanced clustering where the cluster heads are be chosen twice, first by $\mathrm{LEACH}$, and second time by taking into account the node rest energy, but still there problem-consuming energy 
because the protocol chooses $\mathrm{CHs}$ twice. In [9] a new modification for LEACH was presented called Power-Efficient Gathering in Sensor Information Systems (PEGASIS). In this approach, a chain of sensor nodes is formed and randomly chooses the $\mathrm{CH}$, but a dynamic topology was needed to enable the $\mathrm{CH}$ to know the energy status of the other nodes to determine which node is the next in the transmitting data process.

A modification of PEGASIS was presented in [10] which is LEACH-P that combines both LEACH and PEGASIS. It has a dynamic topology for choosing $\mathrm{CHs}$ in a less consuming energy manner. In [11] a new modification of protocols was presented called EB-LEACH which uniformly distributes $\mathrm{CHs}$ all over the network and thus improves the lifetime of WSN, however, the energy between clusters was not balanced in this method. Figure 1 shows the routing in LEACH.

In this paper, a new approach of dividing the network area into clusters is presented by using circular paths to increase the life span of WSN as compared to classic LEACH.

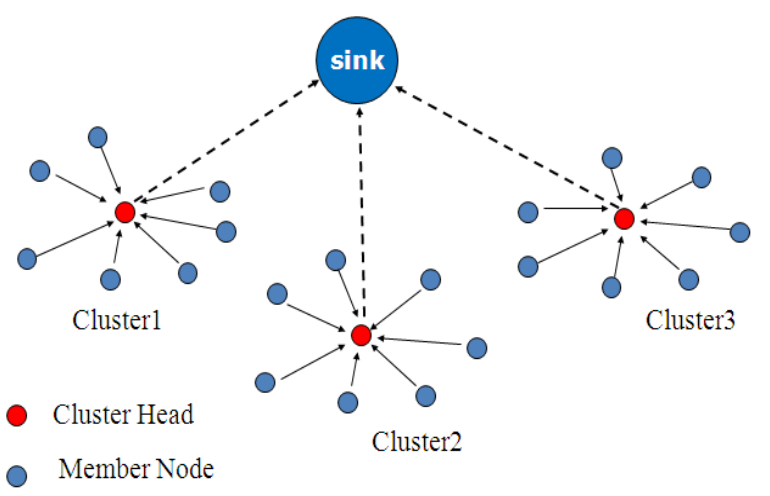

Figure 1. Routing in LEACH

\section{LEACH Protocol}

LEACH protocol is used as a probabilistic formula for the selection of the node in a random way. The tasks of sensing and transmitting the data to the cluster head are performed by a normal node. Whereas, the cluster head performs the duties of processing, aggregation, receiving and transmitting data to the sink or base station. So the cluster head spends more energy than non-cluster head one. Every node will be chosen as a cluster head to continue the process of transmitting data. The consumption of the node's energy will be done systematically until the last node loses its energy and become dead and that will increase the lifetime of the network [12]. LEACH protocol is split into several rounds, each round consists of the following two phases:

\section{Phase 1: Setup}

In this phase, a random number $\mathrm{R}$, from 0 to 1 is selected randomly from each node with a threshold $\mathrm{T}$ value of the node. The threshold can be calculated from the following "(1)":

$$
T(n)=\frac{p}{1-p\left(r\left(\bmod \frac{1}{p}\right)\right)}
$$

Here, $T(n)$ is the threshold value, $P$ is optimal cluster heads selection probability, $r$ is the present round. If the value of sensor node's random number $(R)$ is more than $T$, then the given node will not be appointed as $\mathrm{CH}$ for the current round. If $R$ is less than $T$, the node will be appointed as the $\mathrm{CH}$ for the current round.

After finishing the selection of the cluster heads, the message of the same round propagates to other nodes, which then become the cluster heads. different cluster groups in the network are formed in such a way that the cluster head receives the node request message of high power signal which means that this $\mathrm{CH}$ is closer to the node than other ones. And so on, the distance between the node and the cluster head is minimized and draining of energy in the network is also reduced.

\section{Phase 2: Steady-state}

This phase uses the techniques of CDMA (Code Division Multiple Access) and TDMA (Time Division Multiple Access), therefore, the duration of this phase is longest than the previous one. To obviate the collision between signals, each node has a specific period for transmitting the data, which is assigned from the cluster head. When the data packets received at the cluster head, they aggregate without any confusion using CDMA and move to the sink. in this manner, the first round is finished and the process is repeated until the energy of the remaining nodes is consumed [13].

Figure 2 illustrates the flow chart of LEACH protocol, beginning from the selection of the cluster heads and ending with sending data to the base station.

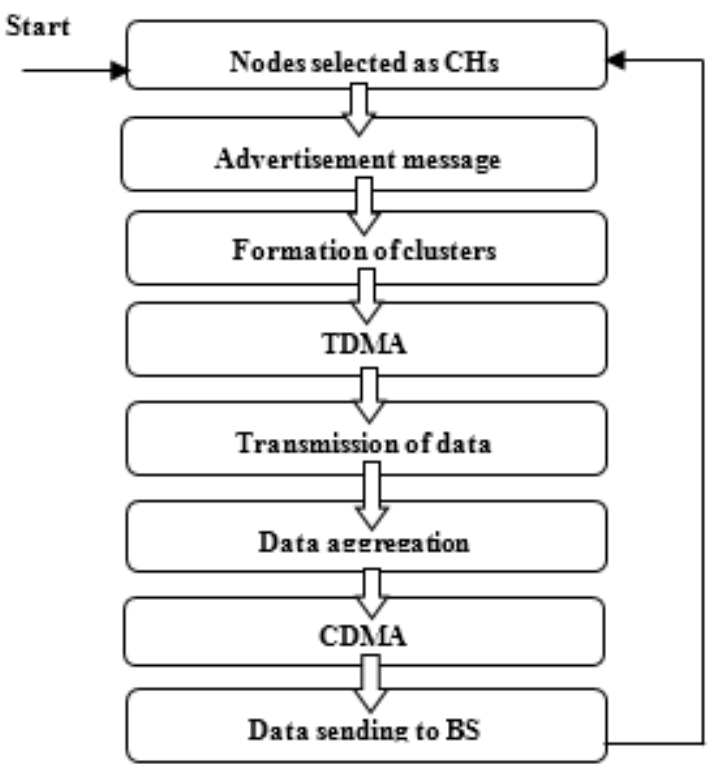

Figure 2. Flowchart of LEACH [12] 


\section{The Proposed I-LEACH (Based on Circular Clustering)}

In LEACH protocol, the cluster heads are distributed randomly, near or far from the base station. The random situated $\mathrm{CH}$ may lead to more energy consumption based on the distance between the $\mathrm{CH}$ and base stations. The number of cluster heads in LEACH is varied in different rounds. Whereas, the improved LEACH [I-LEACH] divides the network into circular clusters where every cluster has its cluster head. The number of cluster heads is an optimal predetermined value.

The following assumptions are made in I-LEACH:

- The energy level of each node in the network can be calculated.

- $\quad$ The base station can receive the messages sent from different nodes in the network.

- The locations of the node can be sent directly from the nodes to the base station.

The proposed I-LEACH is based on traditional LEACH where the rounds are divided into the set-up and steady state periods. In the set-up period, the base station elects the nodes chosen as cluster heads based on their energy level, if the energy exceeds the average energy value. The steady-state period uses the TDMA method for scheduling the data signals sent to the cluster heads and then transmitted to the base station [14].

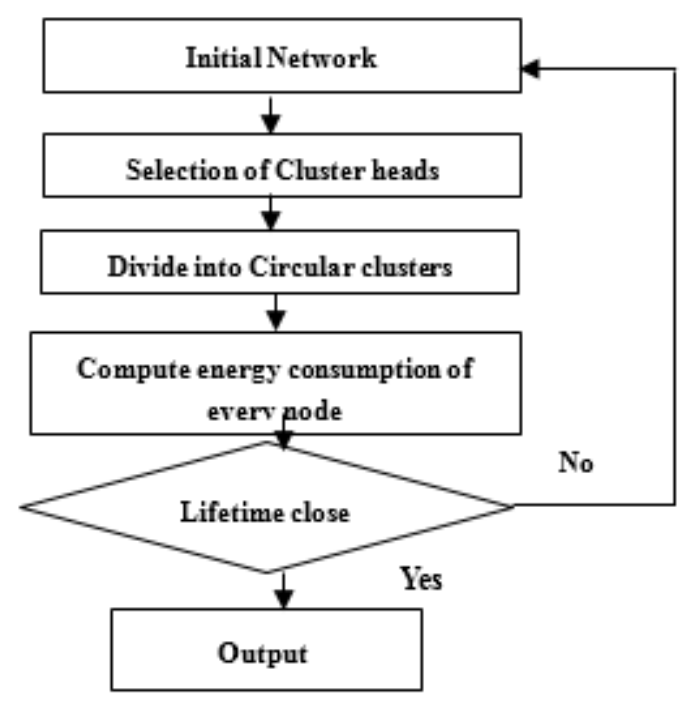

Figure 3. Flowchart of the proposed I-LEACH

The restriction on [circular clusters] is based on the width of the network (W) where W equals 1000 and the diameter of each circular cluster equals the width divided by the number of segments on each side. It will be obvious that the circular clustering will increase the lifetime of the network by organizing the process of sending packets to the base station or sink.

Equations (2) and (3) are the script for the part of drawing circular cluster map in MATLAB

$$
\operatorname{numRx}=\operatorname{fix}\left(\operatorname{sqrt}\left(\mathrm{p}^{*} \mathrm{~N}\right)\right)
$$

Where numRx is the number of segments in each side

$$
\mathrm{dr}=\mathrm{W} / \text { numRx; }
$$

where $d r$ is the diameter of circular clusters.

\section{Simulation and Results}

In this section, the I-LEACH is compared with the classic LEACH. The simulation is carried out using MATLAB. Three cases have been considered in this paper. The parameters of the network are given in the following table 1:

Table 1. Simulation Parameters

\begin{tabular}{|l|l|}
\hline Parameter & Value \\
\hline Network size & $1000 \mathrm{~m}^{2}$ \\
\hline Number of nodes & 200 \\
\hline Initial Energy $\left(\mathrm{E}_{0}\right)$ & $2 \mathrm{~J}$ \\
\hline Cluster head percentage & $0.1 \%$ \\
\hline Number of rounds & 200 \\
\hline Energy for transmitting one bit $\left(\mathrm{E}_{\text {trans }}\right)$ & $1.0000 \mathrm{e}-07$ \\
\hline Energy for receiving one bit $\left(\mathrm{E}_{\text {rec }}\right)$ & $1.0000 \mathrm{e}-07$ \\
\hline Data aggregation energy $\left(\mathrm{E}_{\mathrm{agg}}\right)$ & $1.0000 \mathrm{e}-08$ \\
\hline The energy of free space model amplifier $\left(\mathrm{E}_{\mathrm{fs}}\right)$ & $0.3400 \mathrm{e}-9$ \\
\hline
\end{tabular}

\section{Case 1}

In the first case, the parameters of the network are taken from table 1. The results for the network configuration before and after improvement, the total energy dissipated, the number of alive sensor nodes and the number of throughputs or number of packets is depicted in (Figures 4,5,6,7 and 8), respectively.

Figure 4 shows the network configuration for the classic $\mathrm{LEACH}$, the black bold dots indicate the normal nodes and the black empty dots indicate the dead nodes, whereas, the red ones are the cluster heads (CHs). The blue circle in the middle of the network is for the sink or base station. In this configuration, the cluster heads are chosen randomly whereas the network configuration for the I-LEACH in Fig. 5 separated the network into a number of circular clusters (green circles) and the cluster head is chosen inside each circle only (the red dot).

The total energy dissipated by the classic LEACH is greater than I-LEACH, as shown in fig. 6. Classic LEACH takes 600 seconds to dissipate the total amount of energy while the circular cluster takes 1300 seconds. Moreover, before the time of 300 seconds, the number of dead nodes for the I-LEACH is almost zero, which proves the effectiveness of the proposed I-LEACH as compared to the classic one. The number of throughput or packets sent to the sink node for the circular LEACH is higher than the classic LEACH as shown in figures 7 and 8, respectively. 


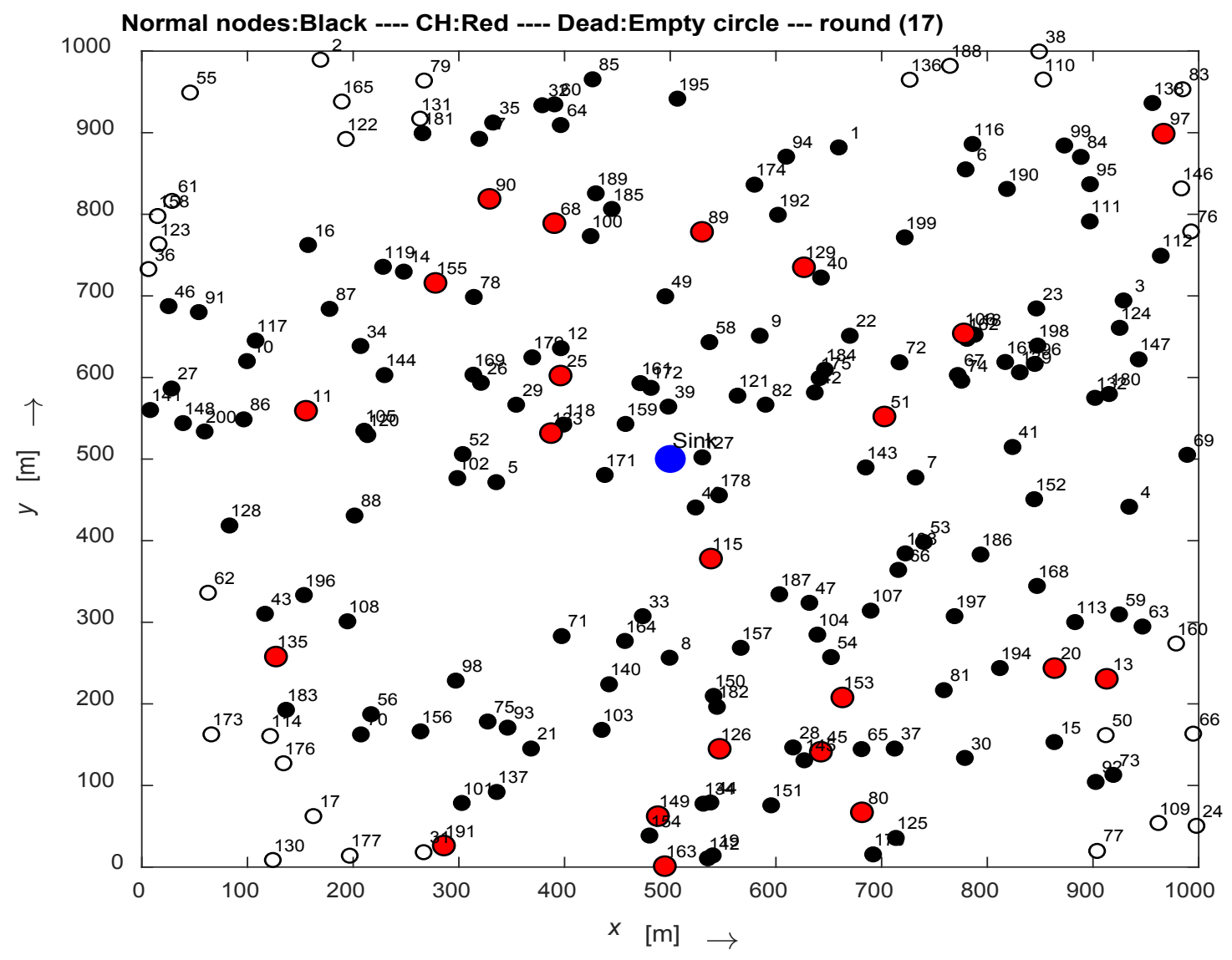

Figure 4. The network configuration for classic LEACH 


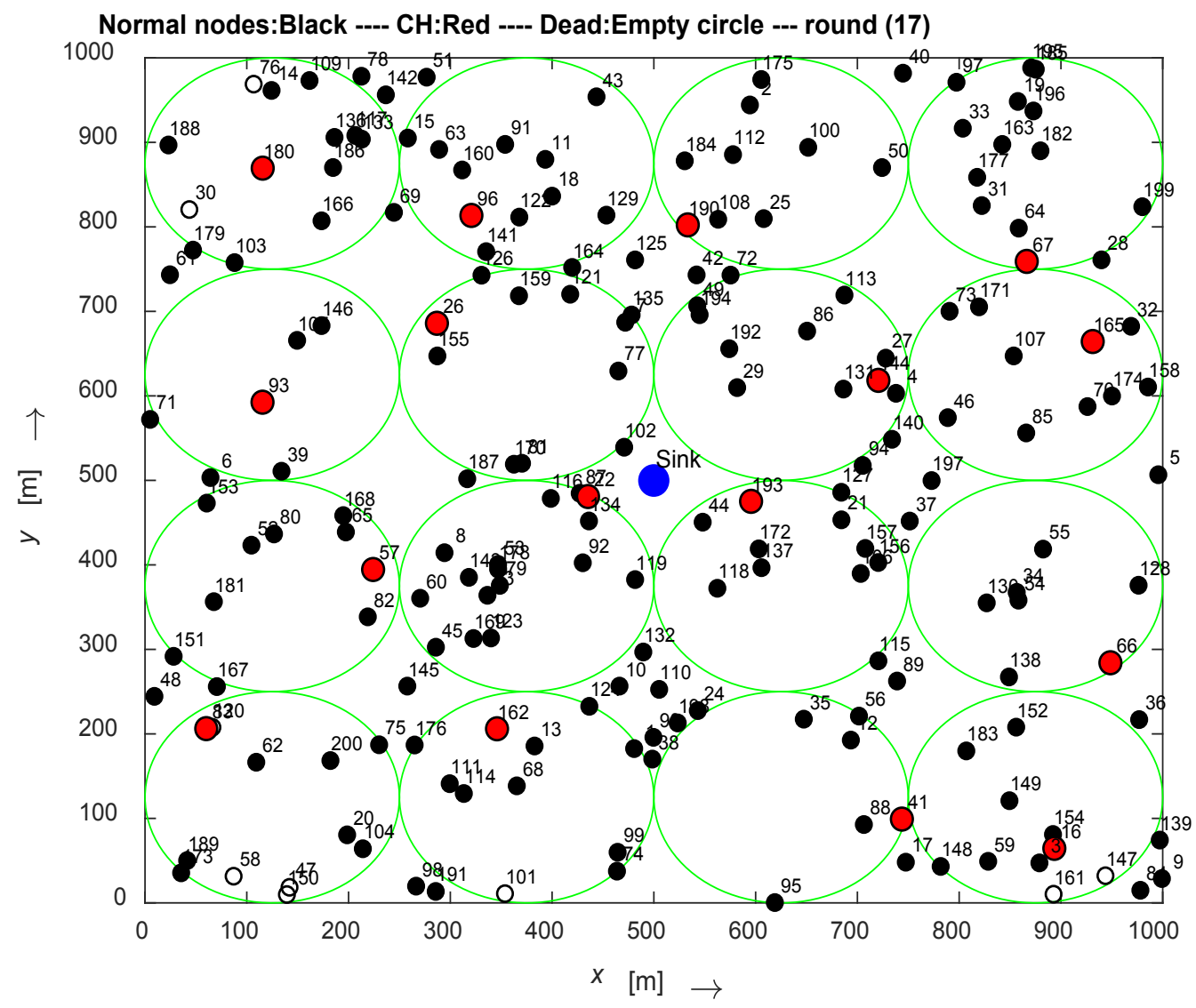

Figure 5. The network configuration for I-LEACH (circular LEACH)

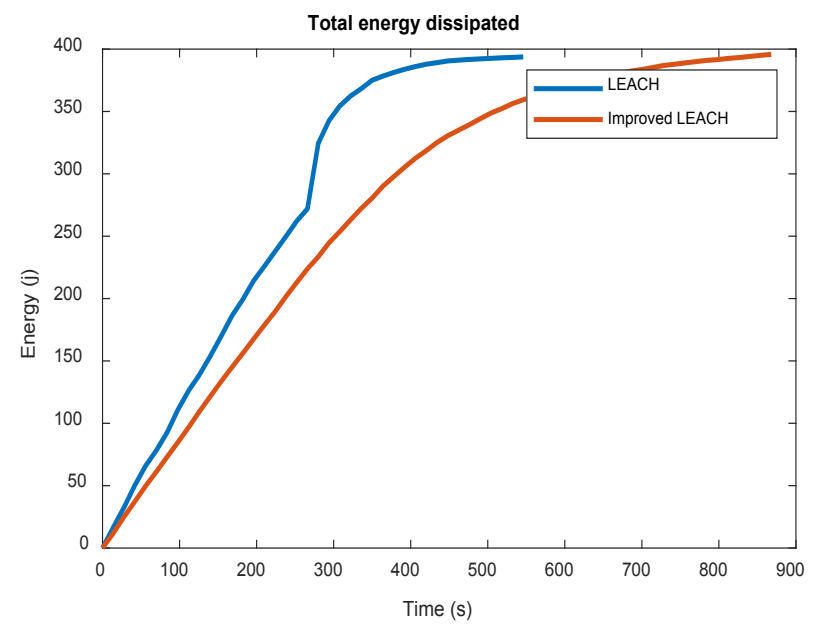

Figure 6. The total energy dissipated versus time.

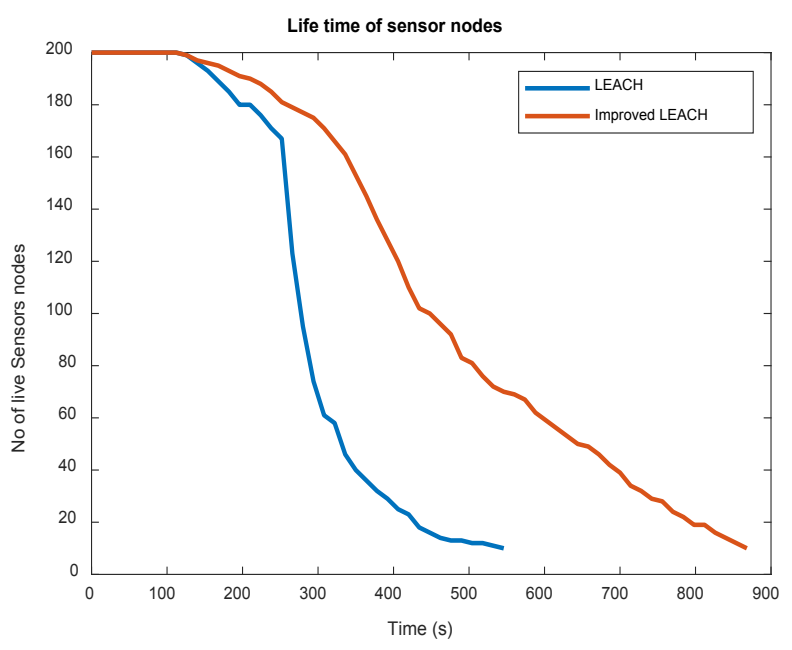

Figure 7. The number of live nodes versus time. 
Case 2

In this case, the number of nodes is increased to 400 node and the remaining parameters are the same as in the first case. As shown in (Figures 8 and 9) the number of dots increased (number of nodes) and the number of green circles also increased with the increasing number of the cluster heads.
The results are shown in figures 10, 11 and 12. The circular cluster is better than the normal one. Energy dissipation is lower and the time taken until the last node becomes dead is more than 2000 seconds. The number of packets sent to the sink node is higher. On the other hand, classic LEACH takes only 1000 seconds until the last node becomes dead and sends only $10 \times 106$ packets to the sink node.

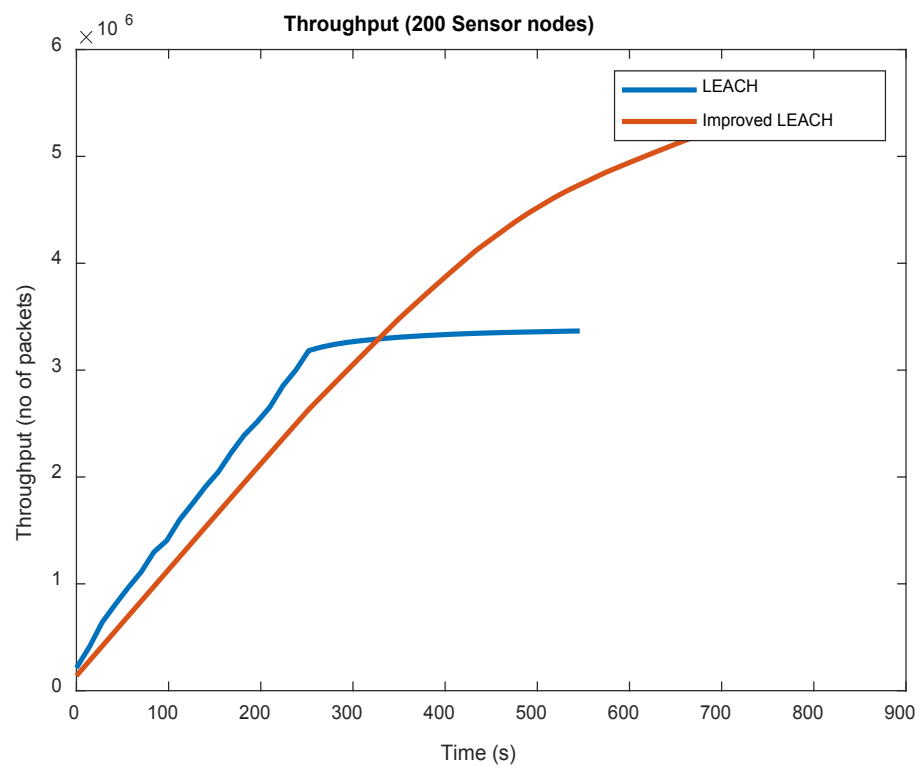

Figure 8. The number of throughputs (Packets) versus time

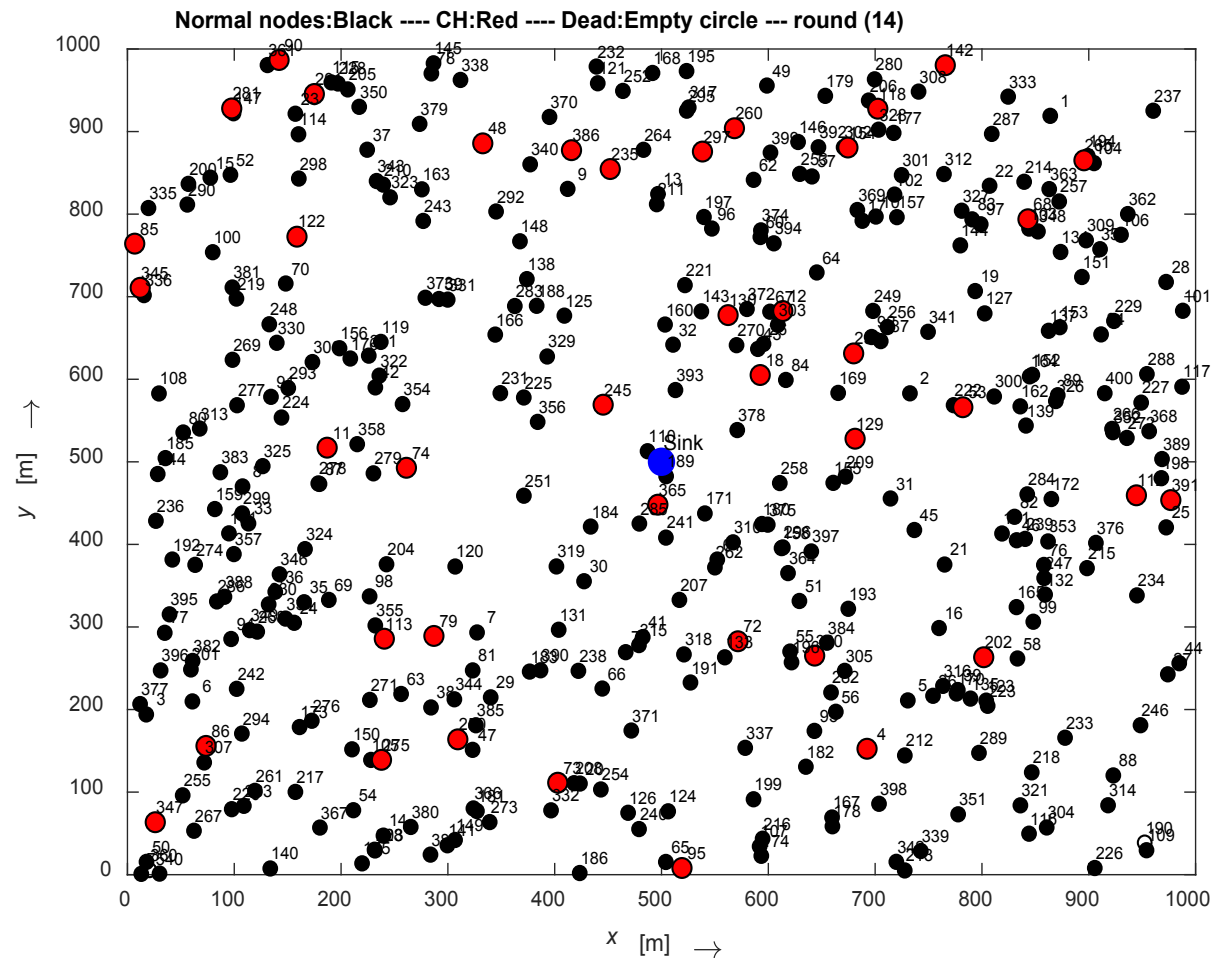

Figure 9. The network configuration for normal LEACH with 400 nodes 


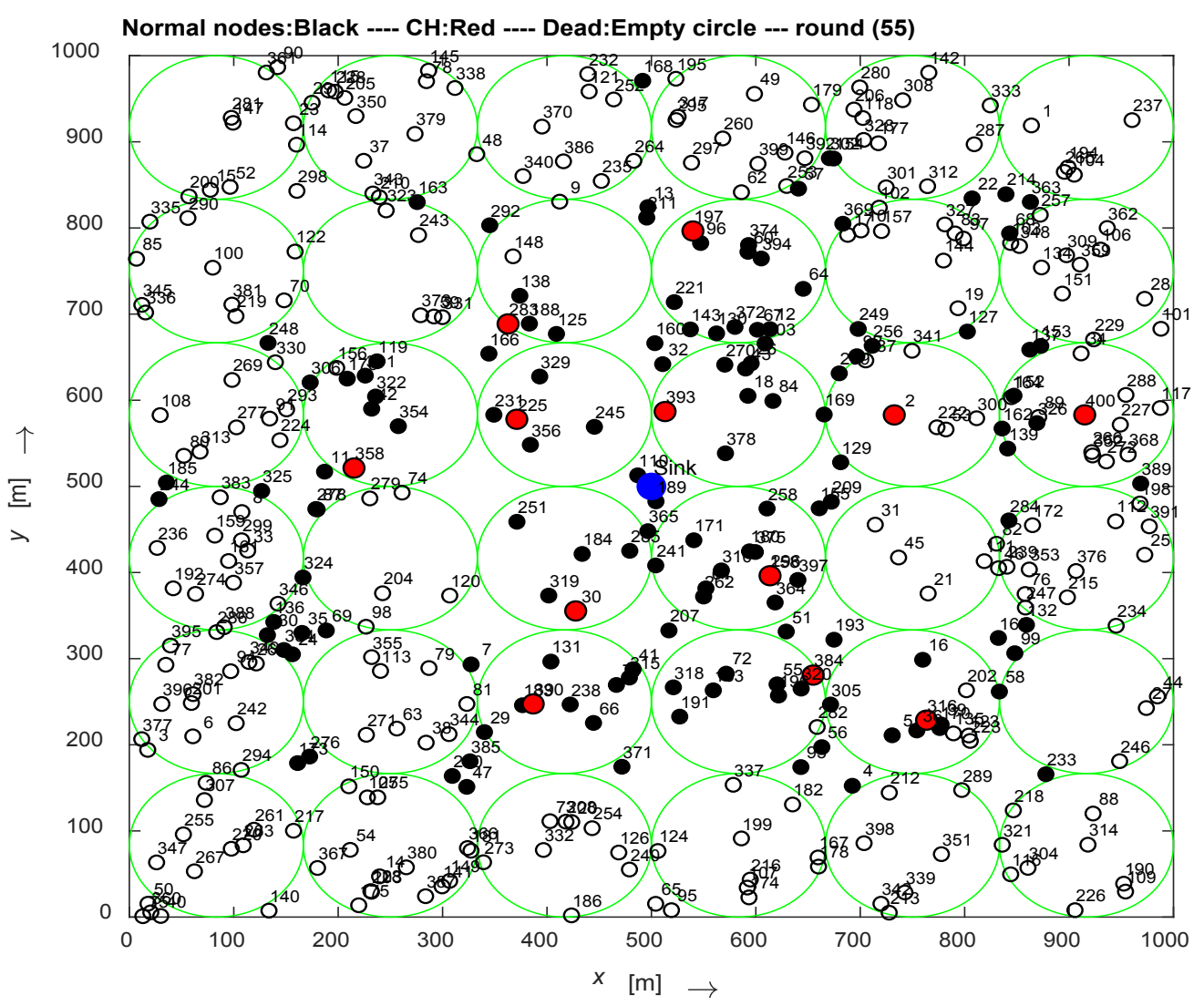

Figure 10. The network configuration for improved Leach (circular LEACH) with 400 nodes

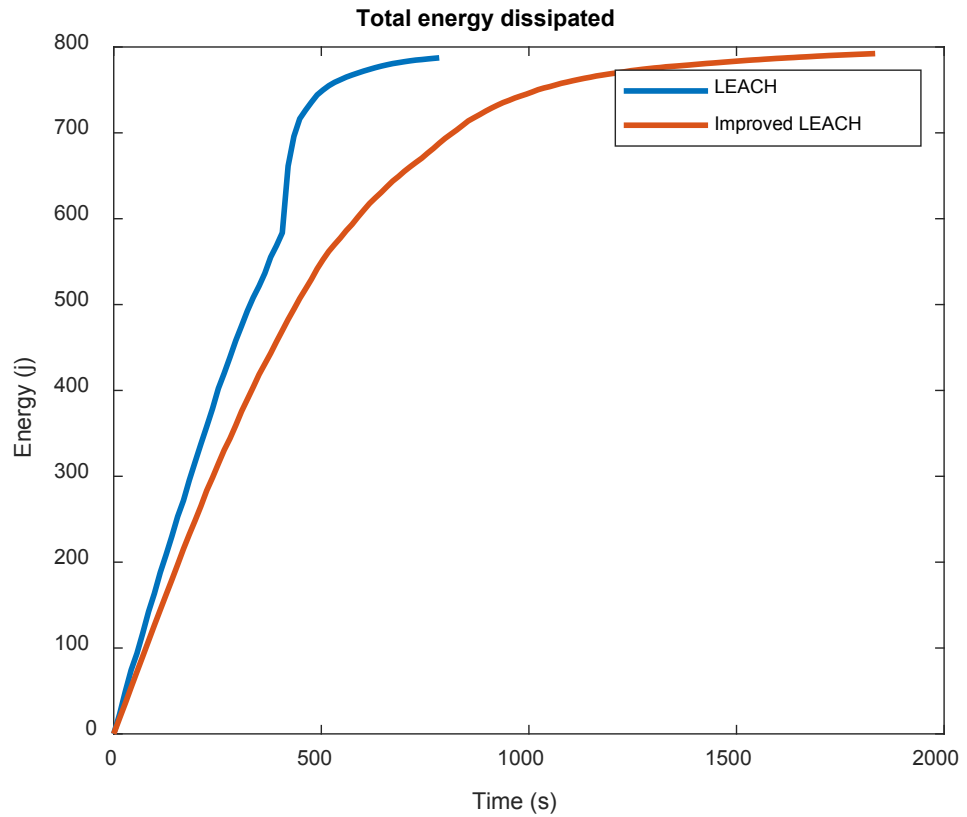

Figure 11. The total energy dissipated versus time with 400 nodes 


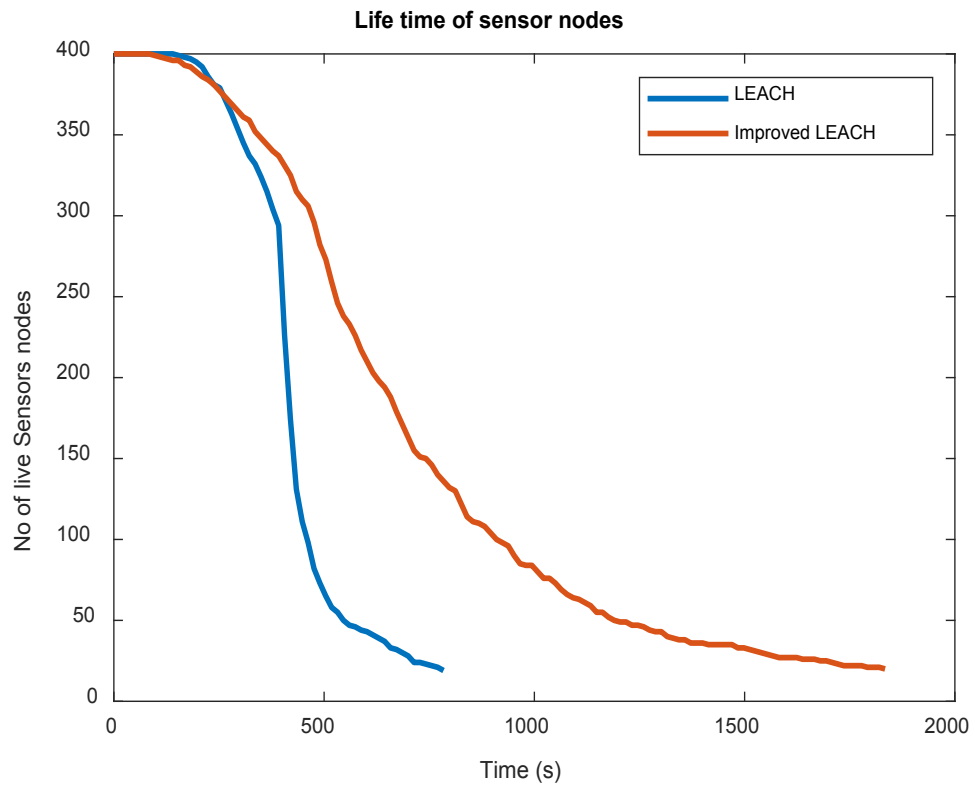

Figure 12. The number of live nodes versus time with 400 nodes

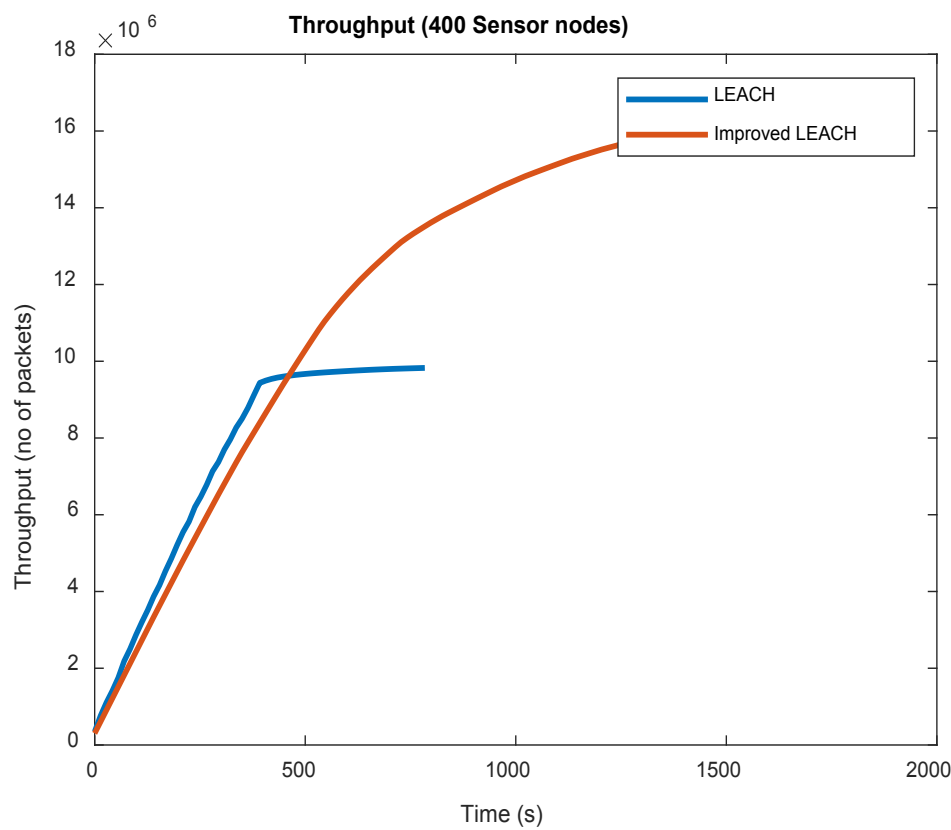

Figure 13. The number of throughputs versus time with 400 nodes

\section{Case 3}

In the third case, the initial energy increased from 2 to 4 Joules and the other parameters are kept the same as case one. The network configurations are the same as in case one since the number of nodes does not change. The other results are shown in (Figures 14, 15 and 16).

When the initial energy of the nodes increases, the time required for the last node to become dead increases also. This is shown in figure 13 where the nodes in the classic LEACH take more than 1000 seconds to dissipate the whole energy while in the case of I-LEACH it takes more than 2200 seconds.

From (Figures 15 and 16) it clear that I-LEACH outperforms the classic one in both the number of live nodes and the number of packets sent to the base station. 


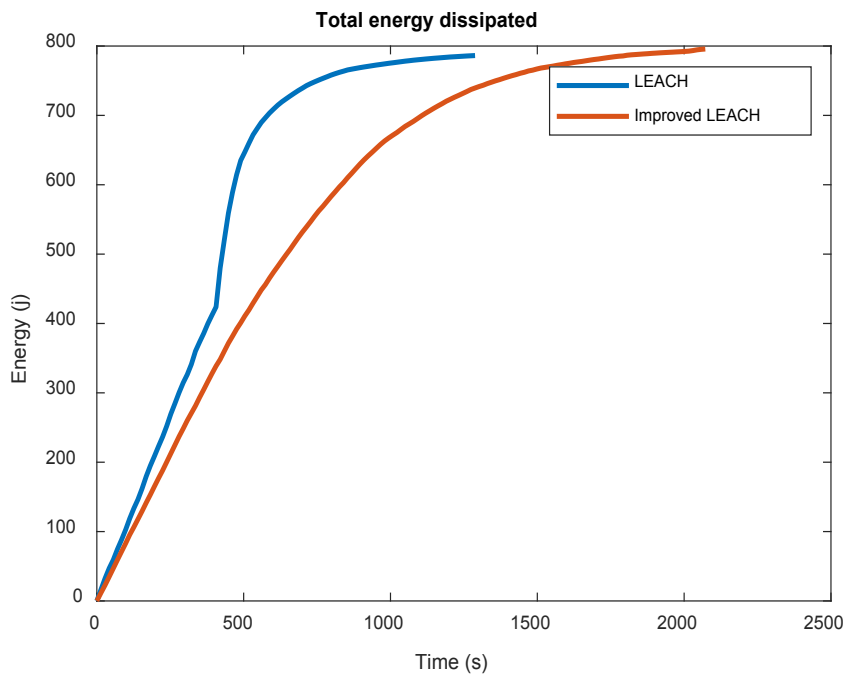

Figure 14. The total energy dissipated versus time at 4 Joules initial energy

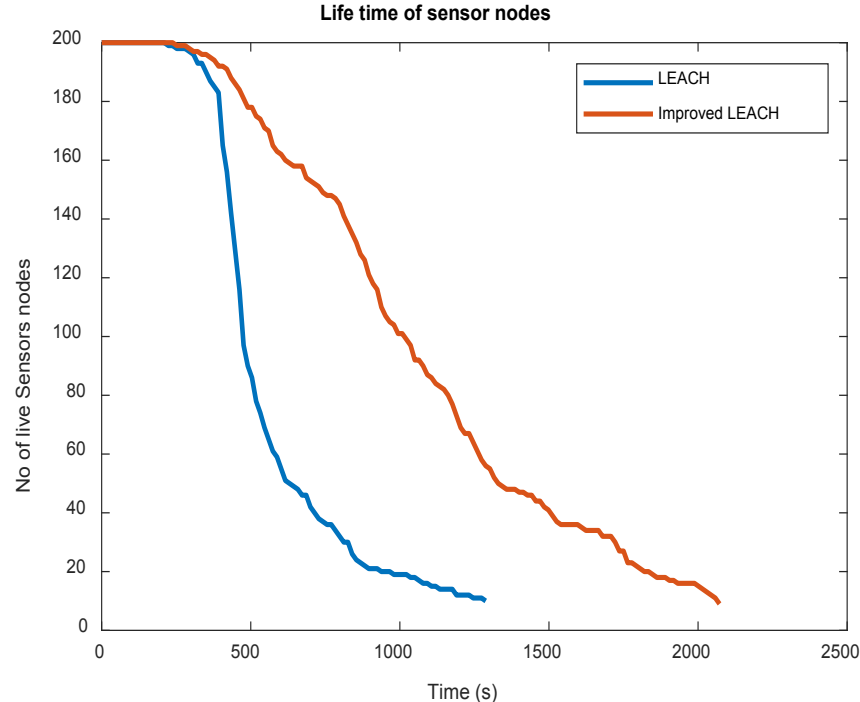

Figure 15. The number of live nodes versus time at 4 Joules initial energy

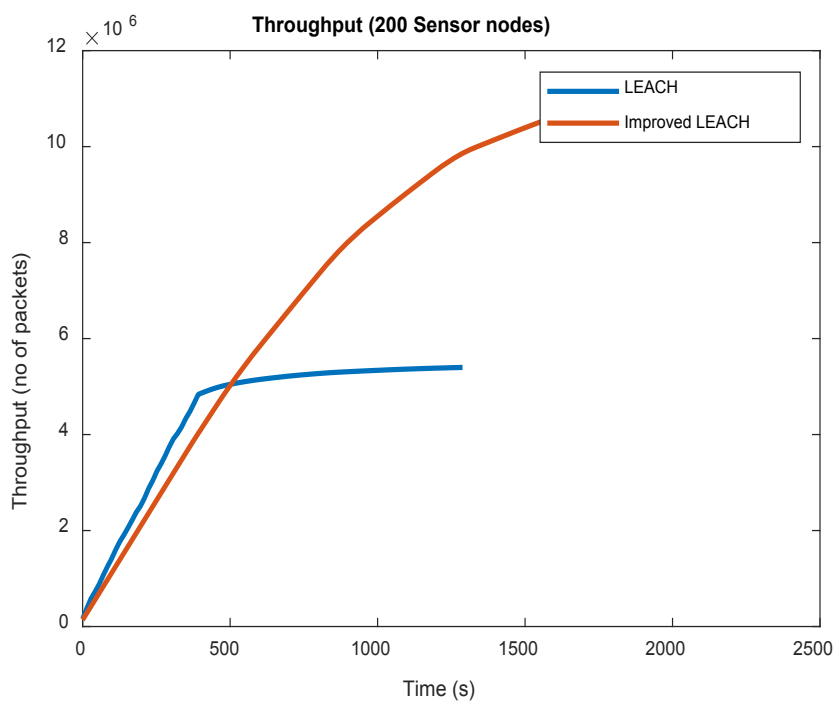

Figure 16. The number of Packets versus time at 4 Joules initial energy 


\section{Conclusions}

In this paper, a new improved LEACH protocol is proposed. The well- known protocol for WSN is called LEACH. It is the first and the most important protocol in WSNs. It uses clusters based distribution methods to transmit data between nodes. The new modification on the LEACH protocol is achieved by dividing the network into clusters. The proposed I-LEACH protocol is superior to classic LEACH in many aspects. From the simulation results, I-LEACH protocol decreases the energy consumption and significantly increases the network lifetime as compared to the original LEACH protocol by decreasing the number of dead nodes and increasing the number of alive nodes through the 200 rounds to continue the process of transmitting data in WSN.

\section{REFERENCES}

[1] R. S. A. Al-Nuaimi. A virtualized routing protocol for improving network lifetime in cluster-based sensor networks. University of Salford, 2017.

[2] I. F. Akyildiz, W. Su, Y. Sankara, and E. Cayirci. A survey on sensor networks, IEEE Commun. Mag., vol. 40, no. 8, pp. 102-114, 2002

[3] K. Ramesh and D. K. Somasundaram. A comparative study of cluster head selection algorithms in wireless sensor networks. arXiv Prepr. arXiv1205.1673, 2012.

[4] C. Intanagonwiwat, R. Govindan, and D. Estrin. Directed diffusion: A scalable and robust communication paradigm for sensor networks, in Proceedings of the 6th annual international conference on Mobile computing and networking, 2000, pp. 56-67.

[5] V. A. Geetha, P. V Kallapur, and S. Tellajeera. Clustering in wireless sensor networks: Performance comparison of leach \& leach-c protocols using ns2, Procedia Technol., vol. 4, pp. 163-170, 2012.

[6] W. R. Heinzelman, A. Chandrakasan, and H. Balakrishnan, "Energy-efficient communication protocol for wireless microsensor networks," in Proceedings of the 33rd annual Hawaii international conference on system sciences, 2000.

[7] W. B. Heinzelman, A. P. Chandrakasan, and H. Balakrishnan. An application-specific protocol architecture for wireless microsensor networks, IEEE Trans. Wirel. Commun., vol. 1, no. 4, pp. 660-670, 2002.

[8] M. Tong and M. Tang. LEACH-B: an improved LEACH protocol for wireless sensor network, in 2010 6th international conference on wireless communications networking and mobile computing (WiCOM), 2010.

[9] R. Mahakud et al.. Energy Management in wireless sensor network using PEGASIS, Procedia Comput. Sci., vol. 92, pp. 207-212, 2016.

[10] A. Razaque, M. Abdulgader, C. Joshi, F. Amsaad, and M. Chauhan. P-LEACH: energy-efficient routing protocol for wireless sensor networks, in 2016 IEEE Long Island Systems, Applications and Technology Conference (LISAT), 2016, pp. 1-5.

[11] H. Mohapatra, S. Debnath, and A. K. Rath. Energy Management in Wireless Sensor Network through EB-LEACH, EasyChair Preprint no. 1192, 2019.

[12] K. Agarwal, K. Agarwal, and K. Muruganandam. Low energy adaptive clustering hierarchy (LEACH) Protocol: Simulation and analysis using MATLAB, 2018 Int. Conf. Comput. Power Commun. Technol. GUCON 2018, pp. 60 64, 2019.

[13] M. U. Javed, Z. Bin Tariq, U. Muneeb, and I. H. Naqvi, Multi-level Dynamic Optimization of Intelligent LEACH with Cost Effective Deep Belief Network, 2019.

[14] I. Sharma, R. Singh, and M. Khurana. Comparative study of LEACH, LEACH-C and PEGASIS routing protocols for wireless sensor network, Conf. Proceeding - 2015 Int. Conf. Adv. Comput. Eng. Appl. ICACEA 2015, pp. 842-846, 2015. 